\title{
Impactos psicológicos da pandemia da COVID-19 na vida de pessoas com tourette
}

\section{The psychological impacts of the COVID-19 pandemic on the lives of people with tourette}

Andréa Adriana da Silva ${ }^{1 *}$, Rodrigo da Silva Almeida ${ }^{2}$, Lirani Firmo da Costa Souza ${ }^{3}$, Neyde Jordânya Gomes Costa de Vasconcelos ${ }^{4}$, Mayara Cristina da Silva Lima ${ }^{5}$

\section{RESUMO}

O presente artigo aborda os impactos psicológicos da pandemia da COVID-19 para a vida de pessoas com Trantorno de Tourette, por meio da metodologia de natureza ensaística. Nesse sentido, o Tourette nada mais é do que uma perturbação neuropsiquiátrica que compromete o sujeito psicossocialmente, causando um intenso sofrimento psíquico às pessoas que o possuem devido às disfunções decorrentes de sua sintomatologia. Além disso, o atual contexto da pandemia tem impactado na saúde mental das pessoas que possuem esta psicopatologia, intensificando ainda mais os danos decorrentes desse quadro clínico. Portanto, difundimos o argumento de que se faz importante o acompanhamento psicológico no intuito de contribuir para uma melhor qualidade de vida e saúde mental destas pessoas.

Palavras-chave: Novo Coronavírus; Tourette; Sofrimento Psíquico, Saúde Mental, Psicologia Clínica.

\section{ABSTRACT}

This article addresses the psychic impacts of the COVID-19 pandemic on the lives of people with Tourette's Trantorno, through an essay methodology. In this sense, Tourette's is nothing more than a neuropsychiatric disorder that compromises the subject psychosocially, causing intense psychological suffering to people who have it due to the dysfunctions resulting from its symptoms. In addition, the current context of the pandemic has impacted the mental health of people who have this psychopathology, further intensifying the damage resulting from this clinical condition. Therefore, we spread the argument that psychological monitoring is important in order to contribute to a better quality of life and mental health of these people.

Keywords: New Coronavirus; Tourette; Psychic Suffering, Mental Health, Clinical Psychology.

\footnotetext{
${ }^{1}$ UFAL - Universidade Federal de Alagoas.*E-mail: andreasilvapsi82@gmail.com

${ }^{2}$ UFS - Universidade Federal de Sergipe

${ }^{3}$ FAVENI - Faculdade Venda Nova Do Imigrante

${ }^{4}$ FAMEESP - Faculdade Metropolitana do Estado de São Paulo

${ }^{5}$ UNIT - Centro Universitário Tiradentes
} 


\section{INTRODUÇÃO}

De acordo com Konkiewitz (2013) o Transtorno de Tourette (TT) consiste num quadro neuropsiquiátrico, com características psicomotoras múltiplas, especialmente tiques verbais e motores. Tais características são bastante evidentes e acarretam diversos prejuízos biopsicossociais tanto para a pessoa acometida quanto os seus familiares. Todavia, é importante destacar que não é uma condição apenas neurológica e psiquiátrica, pois envolve tanto movimentos atípicos, como alterações comportamentais. De acordo com esse autor, é uma patologia difícil de ser compreendida, seja sob a ótica das pessoas acometidas por ela, seja pela perspectiva científica.

Diante disso, acreditamos que refletir sobre os impactos psicológicos da pandemia da COVID-19 para as pessoas que possuem TT é de especial relevância e, que o atual cenário social, histórico e cultural tem impactado na saúde mental da população. Peuker e Almondes (2020), em uma publicação da Sociedade Brasileira de Psicologia (SBP), afirmam que a pandemia do coronavírus constitui-se numa grave situação de calamidade pública que atingiu todas as partes do mundo.

Além disso, a sociedade vem sofrendo notáveis transformações históricas e sociais, reverberando na saúde mental da população gerando diversas consequências psicológicas, como: transtornos de ansiedade, depressão, aumento da incidência de suicídio e automutilação, entre outros. Esses citados, são alguns dos efeitos emocionais que a pandemia tem deixado como marca na maioria das pessoas. No caso do TT, por se tratar de uma patologia, ela prejudica várias áreas da vida, especialmente a psicológica. Nesse sentido, acreditamos que as pessoas com Tourette podem ter a saúde mental ainda mais prejudicada no atual momento da pandemia, uma vez que, em sua maioria, já possuem comorbidades como transtorno de ansiedade generalizada, transtorno obsessivocompulsivo, depressão, etc.

Logo, o presente artigo tem o objetivo refletir sobre os impactos psicológicos da pandemia da COVID-19 para a vida de pessoas com o TT, visto que, há um aumento significativo de casos desse quadro clínico na atualidade, fato este que merece atenção especial. Inicialmente, apresentaremos a metodologia utilizada nessa pesquisa, seguida de um breve histórico sobre o Tourette, dos aspectos sobre sua fenomenologia e suas consequências psicossociais, e por conseguinte, no próximo tópico serão abordados os 
impactos psicológicos da pandemia da COVID-19 para a vida das pessoas com Tourette, e nossas considerações finais.

\section{METODOLOGIA}

A metodologia utilizada nesta pesquisa é de cunho qualitativo, de natureza ensaística. Nesta modalidade metodológica, nosso intuito será refletir, de uma forma crítica e discursiva, a respeito dos impactos psicológicos da pandemia da COVID-19 para a vida das pessoas o TT, onde traremos, com base na literatura, nosso posicionamento sobre este assunto (MINAYO; DESLANDES; GOMES, 2016).

Também assumimos o argumento de Kamler e Thomson (2015) de que a escrita acadêmica não acontece no vácuo, pois assume o caráter dialógico. Isso implica em afirmar que a escrita acadêmica é concebida como integrante de um conjunto de práticas sociais, históricas, culturais e discursivas, sendo o produto do diálogo e da ressonância de outros textos, e por isso possuindo múltiplas possibilidades interpretativas e de utilização.

Consequentemente, a escrita acadêmica não se processa de forma neutra e nem é o simples produto de normas acadêmicas de regras padronizadas de redação e estilo, pois: "[...] as práticas de redação acadêmica produzem ao mesmo tempo um estudioso e um texto. No mundo acadêmico, os textos e seus autores são inseparáveis" (p. 48). Assim, não existe uma separação entre autor e texto, pois ambos formam um conjunto inseparável, imbricamento que é inerente a qualquer tipologia textual, fazendo com o que a escrita esteja diretamente associada a identidade do pesquisador em um determinado contexto social, histórico, cultural e político, caracterizado pela sua singularidade (KAMLER; THOMSON, 2015).

Finalmente, ressaltamos que não é nosso intuito esgotar esta temática e que não visamos trazer soluções definitivas elou respostas prontas para esta problemática. Ao contrário disso, buscamos aqui capturar os principais blocos construtivos existentes sobre este fenômeno e identificar lacunas sobre este assunto e a partir disso trazer reflexões que possam contribuir para a produção de conhecimento e visibilidade para as pessoas que possuem TT (CRESWELL, 2010). A seguir abordaremos um breve histórico sobre o transtorno de Tourette, quesito que consideramos imprescindível para uma maior compreensão dessa patologia. 


\section{UM BREVE HISTÓRICO SOBRE O TRANSTORNO DE TOURETTE}

A primeira descrição de um paciente com tiques e comportamento anormal, foi descrita no ano de 1825, pelo então médico francês Jean Marc GaspardItard, que diagnosticou com "a maldição dos tiques" a Marquesa de Dampierre (ITARD, 1825 apud LOUREIRO et al, 2005). De acordo com Karenine (2012) A Marquesa estereotipava tiques motores e verbais e desde seus sete anos de idade, ela vivia isolada da sociedade por ter comportamento atípico e falar obscenidades.

Já no ano de 1873 Segundo Karerina (2012), Trousseau, relatou à "maladie de tics" com sintomas de palilalia (repetição ou imitação de ecos), tiques motores e verbais. Em 1881, George Beard observou casos de ecolalia (repetição de palavras) e inquietação exagerada. Porém, foi no ano de 1884 que, um aluno de Charcot, George Gilles De La Tourette no Hospital de La Salpêtrière, na França, registrou oito casos de tiques motores e verbais. Tourette recorreu aos antigos casos relatados por Itard e Berad, e chegou à conclusão de que estes casos eram semelhantes aos casos observados por eles, e os agrupou todos em uma mesma categoria.

Assim, como afirma Loureiro et al. (2005) o estudante descreveu uma patologia relacionada a movimentos involuntários, como um distúrbio caracterizado por tiques múltiplos, incluindo o uso involuntário ou inapropriado de palavras obscenas e a repetição involuntária de um som, palavra ou frase de outrem.

Entre outubro de 1885 e março de 1886, Freud freqüentou a enfermaria de Charcot naquele hospital e relatou: "as coréiase, as diversas formas de tiques (por exemplo, a doença de Gilles de la Tourette) estavam recebendo atenção especial durante a época em que frequentou aquele serviço". Acrescentou ainda que "Charcot considerava existirem dois tipos de tiques: o verdadeiro ou neurológico e o histérico. A diferença entre eles é que os verdadeiros são permanentes, enquanto os histéricos desaparecem mais cedo ou mais tarde". Ao final do século XIX e início do XX, com o advento da psicanálise, foram elaboradas hipóteses psicogênicas para o transtorno de Tourette. Estas permaneceram em voga até que os avanços da psicofarmacologia, na década de 50, permitiram a elaboração de hipóteses biológicas (HOUNIE E PETRIBÚ, 1999).

Durante muito tempo o TT foi considerado não apenas como um distúrbio, mas como "um jeito meio nervoso de ser". Personalidades conhecidas como Mozart, um irmão de Tolstoy, e até mesmo Anna Freud viveram de perto o transtorno (HOUNIE E 
PETRIBÚ, 1999). Houve tempos em que o TT era tido como uma maldição, e que quem a tinha estava condenado a manifestar comportamentos bizarros até o fim da vida. É visto ainda hoje, como uma perturbação que causa dificuldades de integração e por vezes, inadaptação dessas pessoas nos vários contextos.

Ultimamente, este tema tem tomado destaque em estudos, pois sua configuração desperta o interesse científico neuropsiquiátrico devido a suas comorbidades com outros transtornos de tiques como é o caso do Transtorno Obsessivo-Compulsivo (TOC) e com transtornos do Neurodesenvolvimento como é o caso do Transtorno de Déficit de Atenção e Hiperatividade (TDAH). A seguir discutiremos a fenomenologia do Tourette.

\section{FENOMENOLOGIA DO TRANSTORNO DE TOURETTE}

O TT trata-se de uma perturbação neuropsiquiátrica com início na infância caracterizada pela presença de tiques motores e vocais (JORGE et al. 2013). Este transtorno antes chamado de Síndrome de Tourette foi alterado para outra classe na construção da nova edição realizada do DSM 5 (2014) passando a integrar o espectro dos transtornos de tiques que, por sua vez, compõe o grupo de transtornos do neurodesenvolvimento.

De acordo com o DSM 5, os tiques são movimentos motores ou vocalizações súbitas, rápidos, recorrentes, não ritmados e que costumam ser vivenciados involuntariamente, mas podem ser suprimidos por períodos de tempo variáveis de forma voluntária. Conforme o manual, o indivíduo pode apresentar vários sintomas de tique ao longo do tempo, mas quando o repertório de tiques retorna é de maneira distinta. Todavia, os tiques podem incluir qualquer grupo muscular ou vocalização, alguns sintomas de tiques são comuns entre grupos de pacientes, como, piscar os olhos ou limpar a garganta.

Sob o ponto de vista fenomenológico, podemos dizer que o tique pode ser descrito como um movimento simples, habitualmente envolvendo pequenos grupos musculares, involuntárias, impossível de ser interrompido quando iniciado e sem fator desencadeante, embora ansiedade o exacerba (ASSUMPÇÃO JR., 2014).

Ainda de acordo com o manual, verifica-se que os tiques são comumente desenvolvidos na infância, no qual tipicamente pode se iniciar entre 4 e 6 anos de idade atingindo o pico da gravidade entre 10 e 12 anos, com um possível declínio na adolescência e na idade adulta, no entanto, um pequeno percentual dos pacientes terão 
sintomas graves ou que pioram na vida adulta. Sendo que a prevalência estimada do TT varia de 3 a 8 a cada 1.000 crianças em idade escolar (DSM 5, 2014).

Os tiques que surgem durante a vida adulta são frequentemente atribuídos a uma reemergência de tiques da infância, pressão e ansiedade fortemente evidente, ou a outros fatores como trauma, acidente vascular cerebral, ou infecção cerebral. Assim, a causa mais comum de tiques de início no adulto é o TT que pode, por sua vez, remitir após a puberdade, mas, expressar-se novamente por tiques intensos vários anos mais tarde, causando comprometimentos significativos não só na esfera biológica como na comportamental, psicológica e social (HOUNIE E MIGUEL, 2012).

É importante salientar, algumas das estereotipias importantes manifestas no TT que caracterizam a doença. Elas são: piscada de olhos, movimentos repentinos com a cabeça, caretas, balanço de ombros, fungada, tosse, sons guturais, gestos obscenos, repetição involuntária de sílabas ou palavras (DSM 5, 2014). Ver Figura 1:

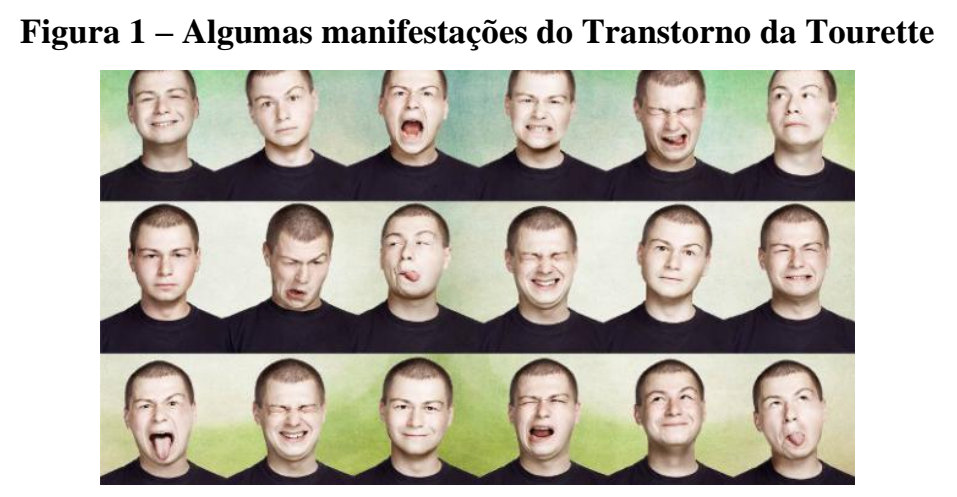

Fonte: (Biblioteca IC, 2016).

Segundo a ASTOC (2016), a anamnese, a história familiar, a observação e o exame neurológico são normalmente suficientes para estabelecer o diagnóstico do transtorno do tique, porém não existem testes diagnósticos confirmatórios. Evidência laboratorial de uma infecção estreptocócica recente pode autorizar a consideração de um tratamento com antibióticos. No presente, estudos de neuroimagem não são diagnósticos, entretanto, têm contribuído para o aumento do conhecimento dos substratos neurobiológicos do Transtorno de Tourette. 


\subsection{Tratamento}

Segundo Karenina (2012), ainda a ciência não encontrou cura para o transtorno de Tourette. Porém, o tratamento é feito a partir da associação das terapias psicossocial e farmacológica, mas é preciso avaliar especificamente os tiques quanto a sua localização, frequência, intensidade, complexidade e interferência, assim como, investigar a vida psicossocial do paciente.

Os tiques, às vezes, incomodam mais as pessoas que estão em volta do paciente do que a ele próprio. Por isso, deve-se considerar qual a alternativa de tratamento a ser utilizada, uma vez que, o uso de fármacos visa à supressão dos tiques ou a redução de sua intensidade, no qual pode ser útil no caso de tiques intensos, pois devido aos efeitos colaterais dos medicamentos, a utilização ficou restrita para pessoas de tiques graves. (HOUNIE; MIGUEL, 2012).

\section{COMPROMETIMENTOS PSICOSSOCIAIS DO TRANSTORNO DE TOURETTE}

De acordo com Konkiewitz (2013) estudos afirmam que paciente com Tourette tem grande probabilidade de terem depressão, transtornos de ansiedade, transtorno explosivo intermitente, transtornos do sono, transtorno opositivo-desafiador, transtorno de conduta, comportamento de auto injúria, transtornos de aprendizado, dentre outros. Bem como, há uma grande incidência de transtorno invasivo do desenvolvimento que é três vezes maior em pacientes com TT, quando comparados à população em geral.

Diante dessas informações podemos dizer que os distúrbios concomitantes geram mais sofrimento e desajuste social que o próprio TT e podem persistir por mais tempo na idade adulta No caso das crianças, o maior prejuízo não reside tanto nos movimentos ou vocalizações, mas sim nos transtornos concomitantes, como TDAH e TOC (KONKIEWITZ, 2013).

Já nos Jovens e adolescentes acometidos, de acordo com Assumpção Júnior (2014), é o transtorno de movimento que mais causa constrangimento, impacto social e emocional. Portanto, esses dados em conjunto reforçam que o TT não pode ser compreendido apenas como um distúrbio de movimento, mas sim como uma alteração neuropsiquiátrica com dimensões afetivas, psicológicas, comportamentais e sociais, uma 
vez, que pessoas com TT possuem dificuldades de interação com outros indivíduos, por serem taxadas de "loucas" e desvalorizadas enquanto pessoas, apesar de possuírem capacidade cognitiva, pensamento lógico e subjetividade como qualquer outro.

\subsection{A subjetividade e o ser/agir no mundo}

A esfera que compõe o Psicossocial é primordial para o indivíduo se encontrar enquanto pessoa, e isto está atrelado intrinsecamente nas relações sociais das pessoas. Silva (2004) afirma que um dos fatores mais importantes e determinantes para a formação da subjetividade de cada pessoa é o convívio social, e na contemporaneidade, que passa por enormes transformações econômicas, sociais, políticas e culturais, se vive um momento histórico intensamente marcado pela internacionalização de conteúdos que estabelece padrões e moldes de ser e viver no mundo. Consequentemente, as relações sociais sofrem modificações e é deteriorada por essas influências que geram exclusão nos que não se encaixam nesses "moldes" e o sujeito, por sua vez, é afetado pela deterioração das relações sociais, devido à expectativa posta em si do "ser perfeito" que afeta diretamente os laços e o convívio social.

Por se tratar de uma patologia que tem seu aparecimento inicial na infância e início da adolescência é possível identificar aspectos que prejudicam a formação da subjetividade não somente pelo comprometimento biológico que causa um certo estranhamento nas pessoas que têm contato com pessoas que têm TT, mas também, pelo social e psicológico do sujeito. De acordo com Silva (2009) a subjetividade é a expressão que diz respeito ao modo de ser, aquilo que particulariza o sujeito e é construída socialmente, numa relação de encontro do eu com o mundo, do "ser-com-os-outros-nomundo".

Assim, de acordo com Myers (2000) O ambiente social em que o sujeito está inserido afeta diretamente sua auto percepção e influencia seu modo de pensar no mundo, bem como, em sua forma de sentir e se comportar. Por isso, em grupos de culturas, raças ou sexos diferentes, notamos como diferimos e como os outros reagem à nossa diferença. Isso sucede porque a preocupação pessoal motiva o comportamento social.

Dessa forma, uma vez que a subjetividade de pessoas com Tourette está substancialmente atrelada ao modo de serem no mundo, podemos refletir que ela abarca as consequências da doença. Assim, pessoas com TT estão constantemente monitorando 
seu comportamento em relação à expectativa do outro, devido às ações involuntárias que são manifestas constantemente. A tensão, o medo e a vergonha são intrínsecos à subjetividade de tais pessoas, que de modo contínuo busca sempre estar se ajustando às expectativas de normalidade do outro.

\section{IMPACTOS PSICOLÓGICOS DA PANDEMIA DA COVID-19 NA VIDA DAS PESSOAS COM TOURETTE}

Em março de 2020, a Organização Mundial da Saúde (OMS) fez a categorização de uma nova doença infecciosa chamada COVID-19 como uma pandemia (doença que rapidamente se espalhou por diversas partes de diversas regiões continental ou mundial). Trata-se de uma doença causada pelo coronavírus, chamado SARS-CoV-2, que apresenta um espectro clínico variando de infecções assintomáticas a quadros graves. Cerca de $80 \%$ dos pacientes com COVID-19 podem ser assintomáticos ou oligossintomáticos (poucos sintomas), e aproximadamente $20 \%$ dos casos requer atendimento hospitalar por apresentarem dificuldade respiratória, dos quais aproximadamente 5\% podem necessitar de suporte ventilatório (WORLD HEALTH ORGANIZATION, 2020).

Essa doença tem infectado pessoas ao redor do mundo, sendo milhares os casos diagnosticados todos os dias. Pelo fato de ainda ser uma doença nova, as autoridades, países e comunidades científicas procuram estudar o com vírus e suas variantes, afim de livrar a população de tal doença que, por sua vez, tem levado outros milhares à óbitos. Esse cenário vivido pela população global tem gerado espanto e principalmente o sentimento de medo da morte nas pessoas que buscam constantemente por informações de sintomas, formas de contágios, prevenção, etc.

Dentre as medidas que têm sido tomadas para o enfrentamento e combate da COVID-19 destacam-se o incentivo ao uso de vacinas, a imposição de medidas de distanciamento social, especialmente o isolamento, a quarentena e o lockdown. O objetivo de tais medidas concentra-se em evitar mortes e novos surtos de novas variantes do vírus o que, por sua vez, inevitavelmente, tem acontecido em diversos países ao redor do mundo, contaminando pessoas, e até mesmo levando sistemas de saúde ao colapso. Assim, acreditamos que essas questões que envolve o cenário dessa nova pandemia no mundo, tiveram importantes impactos para a saúde mental da população em geral, dentre os quais destacamos as pessoas com TT. 
De acordo com Peuker e Almondes (2020) medidas de distanciamento social, apesar de se constituírem como medidas imprescindíveis de saúde pública para o enfrentamento da pandemia, também trazem consigo vários impactos para a saúde mental da população, tendo em vista, que limitam a comunicação e as relações interpessoais face a face, modificando radicalmente a rotina da população. Houve então a necessidade de se utilizar cada vez mais as mídias e ferramentas digitais, seja para trabalhar, seja para ter contato com parentes e pessoas próximas.

Consequentemente, as alternativas remotas começaram a ser utilizadas de forma crescente. Os autores acreditam que a atual crise epidêmica impactou a saúde mental da população de forma intensa, tanto no sentido de adoecer pessoas que até então estavam saudáveis, quanto agravou condições psicológicas preexistentes, aumentando de forma grandiosa a demanda pelos diversos serviços de saúde mental (PEUKER; ALMONDES, 2020).

Em um estudo realizado por Pereira, Medeiros e Bertholini (2020), investigou-se o comportamento dos indivíduos frente ao isolamento social, levando em conta a proximidade de pessoas (amigos, parentes, etc.) vítimas da COVID-19. Em seus resultados, os autores observaram que, à medida que tomam ciência de vítimas fatais, maior é o apoio às políticas de isolamento, diante do "medo da morte" que, por sua vez, tem provocando inúmeras reações emocionais como mecanismo de proteção às suas vidas. Além disso, é importante ressaltar que:

O medo é um mecanismo adaptativo de defesa animal fundamental para a sobrevivência e envolve vários processos biológicos de preparação para uma resposta a eventos potencialmente ameaçadores. No entanto, quando é crônica ou desproporcional, torna-se prejudicial e pode ser um componente-chave no desenvolvimento de vários transtornos psiquiátricos. Em uma pandemia, o medo aumenta os níveis de ansiedade e estresse em indivíduos saudáveis e intensifica os sintomas daqueles com transtornos psiquiátricos pré-existentes (Ornell et al., 2020, p. 232).

Desse modo, diante dos pressupostos apresentados acerca dos danos causados pela pandemia nas pessoas, podemos aqui refletir que pessoas que possuem o TT podem sofrer tais danos psicológicos decorrentes da pandemia, de forma particular e significativa, levando em conta as especificidades da doença. Argumentamos isso pelo fato de pessoas com Tourette terem distúrbios de origem psiquiátrica associados ao problema. 
Segundo os estudos de Bastos (2009) crianças com TT possuem características psicológicas específicas que podem contribuir para estabelecimento de distúrbios psicoafetivos, principalmente no contato com o mundo, no relacionamento interpessoal, e no controle de suas reações emocionais e impulsivas, tanto positivas como negativas. Isto faz com que em situações estressantes, não necessariamente desagradáveis, elas percam não somente o controle de seus movimentos, com a exacerbação dos tiques, mas percam também o controle sobre suas reações emocionais. Também tentam lidar com estes fatores fazendo uso de defesas egoicas de cunho paranoide e obsessivo. Quando não conseguem, se deprimem, ou buscam escapar das dificuldades por meio de reações adaptativas maníacas.

Assim, o TT é tido como uma patologia grave e que afeta o sujeito de forma comprometedora nas mais diversas áreas de sua vida, causando sofrimento psicológico não só nas pessoas que o possui, mas também na família que convive com a doença. Para essas pessoas com TT, o momento atual de pandemia, que engloba incertezas e mortes, faz com que o nível de ansiedade eleve, uma vez que, é frequente que as pessoas com TT já tenham ansiedade. E, como consequência desse pico de ansiedade, tais pessoas sofram uma exacerbação dos tiques de forma intensa e incontrolável o que leva o sujeito a um maior sofrimento psíquico.

Robertson et al. (2020) corrobora esse pensamento quando afirma que a mídia vigente vem estabelecendo um "novo normal", reordenando percepções, gerando mudanças de atitudes, comportamentos e influenciando contextos sociais diante do pessimismo e incertezas sobre a COVID-19.

Desse modo, uma vez que, o confinamento e a quarentena por si só, desencadeiam a ansiedade e gera gravidade dos tiques, é possível que muitas outras anormalidades comportamentais também serão exacerbadas nessas pessoas. Como exemplo temos o TDAH, presente em 50-70\% dos pacientes com TT. Em particular, o fenótipo hiperativo torna o confinamento e a quarentena difíceis de suportar, pois a energia física da pessoa não pode ser liberada. Assim, é provável que ataques de raiva ou explosões, bem como comportamento de oposição possam vir a ser apresentados com intensidade frente ao cenário de pandemia (ROBERTSON et al., 2020).

Além, disso, o TOC é igualmente frequente na TT e diante das instruções governamentais para diminuir e prevenir a contaminação da COVID-19, os pacientes com TOC podem, cada vez mais, vivenciarem conflitos, uma vez que, se orienta o lavar mais 
as mãos, por exemplo, tornando ainda mais obrigante e exaustivo para essas pessoas tal ação. Isso se explica pelo fato deles buscamem constantemente realizar menos essa obra de lavar as mãos, como forma de conter a obsessão. Assim, tal instrução se configura como o oposto do que essas pessoas buscam executar no dia a dia e isso pode resultar em dissonância cognitiva que, por sua vez, pode se tornar quase insuportável para eles (ROBERTSON et al., 2020). .

O autor supracitado ainda coloca que o contexto pode levar a obsessões com a possibilidade de morte de seus familiares ou consigo mesmo o que provavelmente também farão aumentar a ansiedade, possíveis ruminações mórbidas e fenômenos típicos do transtorno causando angustia e mais sofrimento psíquico. Outro pondo que colocado pelo autor, é que as pessoas com TT podem apresentar sintomas socialmente inadequados diante do contexto da crise sanitária e ter tiques de tosse, bem como manifestarem o desejo de apertar as mãos, espirrar, o que dificulta no cumprimento do distanciamento social, e também pode levar o sujeito a mais sofrimento psíquico.

\section{CONSIDERAÇÕES FINAIS}

O TT é uma doença grave e por isso, trata-se de uma patologia neurológica que compromete o sujeito psicossocialmente, e causa intenso sofrimento às pessoas que o possui devido às disfunções psicológicas, sociais e afetivas decorrentes da sintomatologia do transtorno. Com o cenário vigente de pandemia, essas pessoas sofrem não só com as consequências ambientais, mas também com a influência que tais consequências, associadas à doença, acarretam em seu comportamento (exercido de forma sofrível e involuntária ) e em seu psicológico.

Dessa forma, finalmente, argumentamos sobre a importância da ajuda psicológica das pessoas com TT diante da atual pandemia da COVID-19, contemplando a integralidade e diversidade desse público, sendo imprescindível a oferta de atendimento psicológico que tenha como base modelos de intervenção em crise, salvaguardando a proteção individual por meio de orientações para: “[...] evitar o contágio, ajudar a mitigar o sofrimento vivido pelos pacientes e no enfretamento da COVID-19 [...]" (PEUKER; ALMONDES, 2020, p. 4). 


\section{REFERÊNCIAS}

ASSUMPÇÃO JR. F. B. Psiquiatria da infância e da adolescência: casos clínicos. Porto Alegre: Artmed, 2014. ASTOC. Associação Brasileira de Síndrome de Tourette, tiques e transtorno obsessivo compulsivo. In: Associação. Disponível em: <http://www.astoc.org.br/source/php/026.php>. Acesso em 18 Jul. 2020.

BASTOS, A. G.; VAZ, C. E. Estudo correlacional entre neuroimagem e a técnica de Rorschach em crianças com síndrome de Tourette. Avaliação Psicológica. Porto Alegre, v. 8, n. 2, 2009, p. 229-244. Disponível em:

$<$ http://pepsic.bvsalud.org/scielo.php?script=sci_arttext\&pid=S1677-

04712009000200009\&lng=pt\&nrm=iso>. Acesso em 03 Out. 2020.

BIBLIOTECA IC. Imagem Síndrome de Tourret. In: Blog da biblioteca IC. 2016. Disponível em: <https://bibliotecaicfuc.blogspot.com/2016/07/sindrome-detourette.html>. Acesso em: 03 Out. 2020.

CRESWELL, J. W. Projeto de pesquisa: métodos qualitativo, quantitativo e misto. $3^{\text {a }}$ ed. Porto Alegre: Artmed, 2010.

DSM-V. Manual diagnóstico e estatístico de transtornos mentais. $5^{\mathrm{a}}$ ed. Artmed, 2014.

HOUNIE, A. G.; MIGUEL, E. Tiques cacoetes, síndrome de Tourette: Um manual para pacientes, seus familiares, educadores e profissionais de saúde. Porto Alegre: Artmed, 2012.

HOUNIE, A. G.; PETRIBÚ, K. Síndrome de Tourret - revisão bibliográfica e relatos de casos. Revista Brasileira de Psiquiatria, v. 21, n. 1, 1999, p.50-63.

JORGE, J. C.; BARROSO, C.; MONTEIRO, P. O papel da metoclopramida no tratamento da síndrome de Gilles de la Tourette. Acta Pediátrica Portuguesa. Porto, v. 5, n. 44, 2013, p. 265-267.

KAMLER, B.; THOMSON, P. Trabalhando com literaturas. In: SOMEKH, B.; LEWIN, C. (Orgs.). Teoria e métodos de pesquisa social. Petrópolis $\backslash$ RJ: Vozes, 2015, p. 45-55.

KARENINA, S. Síndrome de Tourette: Aspectos psicossociais relacionados ao sofrimento persistente nas famílias que convivem com a doença. Revista RedePsi. 2012. Disponível em: <http://www.redepsi.com.br/2012/08/01/s-ndrome-de-touretteaspectos-psicossociais-relacionados-ao-sofrimento-persistente-nas-fam-lias-queconvivem-com-a-doen-a/>. Acesso em 05 Out. 2020.

KONKIEWITZ, E. C. Tem alguém dentro de mim, tomando o meu lugar: transtorno de tourette. In: KONKIEWITZ, Elisabete Castelon (Org). Aprendizagem, comportamento e emoções na infância e na adolescência: uma visão transdisciplinar. Dourados-MS: Editora UFGD, 2013. p. 125-146. 
LOUREIRO, N. I. V. et al. Tourette: por dentro da síndrome. Revista Psiquiátrica Clínica. vol.32, n.4, 2005, pp.218-230. Disponível em:

<http://dx.doi.org/10.1590/S0101-60832005000400004>. Acesso em 28 Set. 2020.

MINAYO, M. C. S.; DESLANDES, S. F.; GOMES, R. (Orgs.). Pesquisa social: teoria, método e criatividade. Petrópolis: Vozes, 2016.

MYERS, D. G. Psicologia Social. Rio de Janeiro: LTC - Livros técnicos e científicos. Editora: S.A, 2000.

ORNELL, F. et al. Medo pandêmico" e COVID-19: carga e estratégias de saúde mental. Brazilian Journal Psychiatry. v. 42, n. 3, 2020, p.232-235. Disponível em: $<$ http://www.bjp.org.br/details/943/en-US/-pandemic-fear--and-covid-19--mentalhealth-burden-and-strategies>. Acesso em: 09 Out. 2020.

PEREIRA, C.; MEDEIROS, A.; BERTHOLINI, F. O medo da morte flexibiliza perdas e aproxima polos: consequências políticas da pandemia da COVID-19 no Brasil. Revista Administração Pública. Rio de Janeiro, v. 54, n. 4, 2020 p. 952-968. Disponível em: $<$ http://www.scielo.br/scielo.php?script=sci_arttext\&pid=S0034$76122020000400952 \& \operatorname{lng}=$ en\&nrm=iso $>$. Acesso em 10 Out. 2020.

PETRIBÚ, K.; HOUNIE, A. G. Síndrome de Tourette - revisão bibliográfica e relato de casos. Revista Psiquiátrica Brasileira. São Paulo, v. 21, n. 1, 1999, p. 50-63.

Disponível em: <http://www.scielo.br/pdf/rbp/v21n1/v21n1a10.pdf>. Acesso em: 29 Set. 2020.

PEUKER, A. C.; ALMONDES, K. M. Recomendações para o exercício profissional presencial e on-line da psicologia frente à pandemia de COVID-19. Sociedade Brasileira de Psicologia, 2020. Disponível em: <http://www.sbponline.org.br/enfrentamento-covid19\&gt>. Acesso em 08 Set. 2020.

PIROLA, S. M. F.; FERREIRA, M. C. C. O problema da indisciplina dos alunos: Um olhar para as práticas pedagógicas cotidianas na perspectiva de formação continuada de professores. Revista Olhar de Professor. Ponta Grossa, v. 10. n. 2. 2007, p. 81-99.

ROBERTSON M. M. et al. Gilles de la Tourette Syndrome: advice in the times of COVID-19. Rev. Opinion article. In: F1000 Research, 2020, Disponível em: <https://doi.org/10.12688/f1000research.23275.2>. Acesso em 8 de Out. 2020.

SAWAIA, B. (Org.) As artimanhas da exclusão. Petrópolis/RJ: Vozes, 2001.

SCOPEL, L.; RUDNICKI, T. Intervenções cognitivo-comportamentais no atendimento infantil: relato de uma experiência. Saúde e Desenvolvimento Human, v. 3, n. 2, 2015, p. 147-153. Disponível em: <file:///C:/Users/Kelly/Downloads/2432-8296-1-PB.pdf>. Acesso em: 08 Out. 2020.

SILVA, E. F. G.; SANTOS, S. E. B. O impacto e a influência da mídia sobre a subjetividade. Faculdade do Vale do Ipojuca - FAVIP, 2009. 
SILVA, M. V. O. (Org.). Psicologia e direitos humanos: subjetividade e exclusão.

São Paulo: Casa do Psicólogo, Brasília, DF: Conselho Federal de Psicologia, 2004.

WORLD HEALTH ORGANIZATION. Oxygen sources and distribution for COVID-19 treatment centres: interim guidance. In: World Health Organization, 2020. Disponível em: <https://apps.who.int/iris/handle/10665/331746>. Acesso em 03 Out. 2020.

ZILIO, R. P. Terapia Cognitivo Comportamental no Tratamento da Síndrome de Tourette. Dissertação (Mestrado em Psicologia) - Universidade Paulista, São Paulo, 2017. Disponível em: <https://pt.scribd.com/document/348445380/Terapia-CognitivoComportamental-no-Tratamento-da-Sindrome-de-Tourette>. Acesso em 2 Set. 2020.

Recebido em: 2022

Aprovado em: 2022

Publicado em: 2022 